\title{
ACKNOWLEDGEMENT TOREVIEWERS
}

Over the past year the following individuals have assisted in the production of Pathology by reviewing manuscripts. Their time and expertise are gratefully acknowledged.

\begin{tabular}{|c|c|c|c|c|}
\hline N. Acott & Y. Y. Cheng & C. Farah & P. Но & J. Lasota \\
\hline R. Adams & A. Cheong & G. Farshid & A. Hofmeyr & K. W. Lau \\
\hline K. Aivazian & R. Chetty & E. Favaloro & C. Hogan & C. S. Lee \\
\hline A. Al-Ibraheemi & K. L. Chew & J. Felix & G. Hogg & S-H. Lee \\
\hline R. Ameratunga & L. Chirieac & L. Fennell & J. Holland & V. K. M. Lee \\
\hline L. Anderson & R. Chiu & J. Ferguson & K-J. Hsiao & K. Leite \\
\hline A. Antonsson & A. Chou & P. Ferguson & L. Hueston & C. Leslie \\
\hline M. Arbyn & W-C. Chou & D. Fernandez-Nieto & Y. Hung & M. Leung \\
\hline P. Argani & K. Chua & K. Filipski & R. Hurdayal & C-F. Li \\
\hline J. E. Armes & K. L. Chuah & C. D. M. Fletcher & S. Hyne & C. Lim \\
\hline S. Asa & K. Chutipongpisit & J. Fletcher & H. Iland & H. Y. Lim \\
\hline K. Asadi & V. Clifford & C. Florkowski & D. Ingle & M. H. T. Lim \\
\hline S. Badve & A. Clouston & L. Fox & K. Ireland-Jenkin & M. J. Lim \\
\hline T. Baillie & V. Cogger & G. Francis & I. Irving & R. Lin \\
\hline R. W. Baird & A. Colebatch & M. Fritzler & A. Iyer & C. Liu \\
\hline R. Balleine & J. Collett & D. Fulcher & W. C. Jacobs & M. Locketz \\
\hline C. Barrera & L. Collins & L. Furfaro & A. Jennison & T. P. Loh \\
\hline T. Beer & M. Cook & R. Garcia & J. Joseph & J. Lokan \\
\hline D. Bell & G. Coombs & R. Gasiorowski & N. Joseph & R. Lourie \\
\hline B. Bennetts & C. Cooper & A. Gibello & S. Juneja & Z. Lu \\
\hline M. Bettington & K. Cooper & J. Gibson & P. Kalita de Croft & L. Ma \\
\hline P. Bhattacharyya & A. M. Costa & A. Gifford & K. Kaplan & S. MacCallum \\
\hline T. Biagioli & D. Creytens & A. Gill & R. Karim & M. L. Marques-Piubelli \\
\hline T. Blackmore & P. Crispin & D. Gillis & K. Kasem & G. Martignoni \\
\hline M. Bloomfield & G. Cross & E. Glassy & C. Keane & C. Martin \\
\hline P. Blombery & K. Crotty & G. Gobe & P. Keating & N. Masir \\
\hline S. Boers & M. Cummings & D. Govender & G. Kelsey & M. May \\
\hline F. Bonar & C. Cuomo & N. Graf & J. Kench & G. McAuliffe \\
\hline X. Bossuyt & I. da Silva & M. Graham & T. B. Keng & G. McCluggage \\
\hline P. Britton & S. Dahiya & T. Gray & A. Kesson & S. McCormick \\
\hline I. Brown & J. Daly & R. Greaves & T. Y. Khong & C. McIver \\
\hline C. Bryant & A. Datta Mitra & S. Grebe & T. Khor & P. McKelvie \\
\hline C. Bundell & M. Davies & G. Grigoriadis & S. Kidd & J. McKenney \\
\hline A. Burke & R. Davis & M. Grimm & T. Kidd & C. McKenzie \\
\hline K. Busam & B. De Boer & P. Groenen & K. Kikuchi & P. McKenzie \\
\hline D. Cao & A. de la Fouchardiere & A. Gru & M. Killingsworth & C. A. McLean \\
\hline A. Carter & V. De Petra & T. Gulholm & K-Y. Kim & L. J. Medeiros \\
\hline A. Caswell & B. Delahunt & D. Gunawardane & R. Kirsch & K. Meehan \\
\hline C-Y. Chai & B. Dessauvagie & M. Gupta & S. Klebe & N. Mesbah Ardakani \\
\hline S. M. Chai & W. Dimech & F. Haller & J. Knox & C. Miles \\
\hline I. Chambers & A. Dodds & N. Hamad & D. Koch & G. Miller \\
\hline R. Chambers & J. Douglass & P. Harris & K. Kochan & R. Miller \\
\hline A. K-Y. Chan & C. Dow & D. Hartman & T. H. Koh & M. Mino-Kenudson \\
\hline M. Chan & L. Doyle & I. Hashim & K. Konstantinos & C. Mitchell \\
\hline R. Chan & J. D’Rozario & D. Hawkes & B. Koszyca & D. Moffat \\
\hline K-C. Chang & J. Druce & C. Hawkins & G. Kotsiou & P. Mollee \\
\hline A. Charles & E. Duhig & C. Heath & G. Kristiansen & G. Monteuuis \\
\hline A. Charlton & L. Dunk & J. Hecht & H. Kua & E. Montgomery \\
\hline D. Chatterjee & J. Eble & R. Hegele & B. Kumar & C. A. Moran \\
\hline A. Cheah & L. Egevad & C. Hemmings & P. Kumarasinghe & S. Morgan \\
\hline C. Y. Cheah & D. El Demellawy & A. Hendricks & A. K. Y. Lam & A. Morris \\
\hline P-L. Cheah & P. Emanuel & S. Hendry & C-W. Lam & O. Morrissey \\
\hline R. Chean & R. Emerson & A. Henwood & D. Lambie & A. Muljono \\
\hline L. Chee & A. Evans & P. Hickman & R. Langer & S. Munakata \\
\hline L. Cheng & P. Fairweather & A. Hida & N. Langlois & Z. Naing \\
\hline
\end{tabular}




\begin{tabular}{|c|c|c|c|c|}
\hline F. Nakamura & P. Qi & B. Seo & K. B. Tan & A. Verrall \\
\hline H. Nandurkar & X. Qin & H. Sheorey & P. H. Tan & W. Wang \\
\hline T. Nester & D. Rabbolini & M. Sheppard & Y. E. Tan & D. Waring \\
\hline J. Newcombe & D. Ralph & J-S. Shin & G. Taylor & P. Waring \\
\hline P. Newton & S. Ramirez & L. Sholl & P. Taylor & A. Wei \\
\hline H. K. Ng & V. Rathi & K. Sikaris & N. Tee & R. Weinkove \\
\hline J. Nicholls & R. Rawson & V. Sintchenko & E. Tegg & G. Weldhagen \\
\hline K. Nicholls & W. Raymond & V. Sivasubramaniam & B. Thakral & D. Westerman \\
\hline R. Norton & J. Reed & A. Skene & T. Theis & R. Whan \\
\hline M. Nissen & P. Renaut & J. L. Slavin & M. Thunders & B. Wilcken \\
\hline B. O'Brien & S. Riminton & E. Smit & A. Tie & R. Willemze \\
\hline E. Oliva & A. Roberts & D. Smith & I-S. Tiong & R. A. Williams \\
\hline K. M. O’Sullivan & J. Robertson & D. W. Smith & K. F. To & S. Wills \\
\hline A. Osunkoya & J. Robson & C. Snell & J. Tobin & M. J. Wilsher \\
\hline M. Page & A. Roden & J. Solomon & B-H. Toh & D. Wong \\
\hline C-C. Pan & M. Rodriguez & G. Somers & G. Tozbikian & K. K. Wong \\
\hline K. Papanaoum & G. Rossi & A. Sorokin & R. J. Trent & R. W-C. Wong \\
\hline S. Parsons & C. Rosty & D. Spagnolo & M. Tretiakova & B. A. Wood \\
\hline S. Partridge & L. Roth & M. Spellerberg & G. M. Tse & D. Wright \\
\hline R. M. Patel & D. Roytson & A. Srivastava & J. Turnidge & R. G. Wright \\
\hline D. Payton & P. Russell & J. Srigley & J. Tye-Din & $\mathrm{H}-\mathrm{T} . \mathrm{Wu}$ \\
\hline F. Petersson & P. A. Russell & J. Stanek & G. Tyson & S-Y. Xiao \\
\hline Y. S. Piao & A. Ruszkiewicz & M. Staples & K. Uehara & M. Yakin \\
\hline C. Pitman & M. Sait & A. Stevens & A. Upton & R. Yamaguchi \\
\hline Y. Polar & R. Salgado & W. Stevenson & T. van der Kwast & M. Yan \\
\hline A. Pollett & C. Salinas-La Rosa & C. Stewart & C. Vargas & T. Yang \\
\hline O. Prall & H. Samaratunga & P. Stewart & W. Varikatt & P. Yarrow \\
\hline G. Price & L. Satgunaseelan & C. Streutker & M. Varma & I. Yeh \\
\hline C. Prieto-Granada & Y. Sato & E. Sumithran & S. Vasikaran & D. Yeung \\
\hline N. Prosser & A. Schlosberg & J. Sy & V. Vaska & L. Ying \\
\hline E. Provenzano & A. Scott & K. Talia & J. Veinot & M. Yong \\
\hline C. Przybycin & J. Scurry & B. Tallon & E. Verner & Z. Zaini \\
\hline
\end{tabular}

